\title{
Notes
}

\section{On the Solution of Troesch's Nonlinear Two-Point Boundary Value Problem Using an Initial Value Method}

This paper treats the nonlinear, two-point boundary value problem formulated by Weibel [1] and Troesch [2], which is to solve the nonlinear differential equation,

$$
d^{2} y / d x^{2}=n \sinh (n y), \quad n>1,
$$

subject to the boundary conditions

$$
\begin{aligned}
& y(0)=0.0 \\
& y(1)=1.0 .
\end{aligned}
$$

As was pointed out by Troesch [2] and Ehrlich [3], this two-point boundary value problem is inherently unstable and difficult. Roberts and Shipman [4] suggested that this equation can be solved by the combination of three methods, namely, the perturbation technique, the parallel shooting method, and the continuation method. This combination of methods is necessary since none of these methods by itself is sufficiently potent. This method gives accurate solutions of Eq. (1) for $n<5$. For $n \geqslant 5$, however, their results do not converge to sufficient accuracy. Subsequently, Jones [5] used a modified Newton method for the solution of this problem. Even though accurate solutions are obtained for large values of $n$, the number of iterations is increased considerably. As an example, the number of iterations for $n=10$ is 53. Most recently, Miele, Aggarwal, and Tietze [6] applied a modified quasi-linearization method in which the interval of integration is subdivided into a number of subintervals. While the number of iterations is greatly reduced, the number of subintervals is large for large values of $n$. To demonstrate this point, let us again consider the solutions for $n=10$. Accurate solutions are obtained with only 9 iterations, which represent a significant improvement over Jones' [5] 53 iterations. However, the interval of integration has to be divided into as many as 18 intervals and, for each subdivision, 100 integration steps are needed. It therefore requires a total of 16,200 integration steps (for 9 iterations) to obtain the solution for $n=10$.

In this paper, a noniterative method, known as the method of transformation groups, will be applied for the solution of this problem. The method is simple and less time-consuming, and the results are accurate. 
Two-point boundary value problems are very common in various disciplines of applied science and engineering. Traditional methods of solving this type of equations rely heavily on the so-called Shooting Technique or its modifications. In a shooting method, the missing initial condition is assumed and the differential equation is integrated as an initial value problem until the independent variable reaches the terminal point. The calculated terminal condition is then compared with the given terminal condition. If they agree within a certain specified degree of accuracy, the assumed value is the correct answer. Otherwise, another value has to be selected (in some systematic way). This iterative approach is sometimes timeconsuming and may run into difficulty of convergence due to the special characteristics of the problem, such as that of Eq. (1) as discussed in [1-6]. The method of transformation groups is a very effective alternative method of solving this kind of problems in that the method is noniterative. It was initiated by Töpfer [7] and extended by Klamkin [8] and $\mathrm{Na}[9,10]$. By this method, the original two-point boundary value problem is first converted to an initial value problem using a transformation. The required initial condition of this equation is determined in the process of transformation. This initial value problem can be solved by any numerical integration scheme. By transformation back to the original variables, the solution of the original nonlinear boundary value problem is obtained. The entire process is relatively fast and the results are found to be accurate.

To solve Eq. (1) by this noniterative method, let us introduce a new dependent variable,

$$
z=n y .
$$

Equation (1) and its boundary conditions then become

$$
d^{2} z / d x^{2}=n^{2} \sinh z,
$$

subject to the boundary conditions

$$
\begin{aligned}
& z(0)=0, \\
& z(1)=n .
\end{aligned}
$$

To reduce the problem into an initial value problem, let us introduce the socalled linear transformation as

$$
x=\bar{x} A^{\alpha_{1}}, \quad z=\bar{z} A^{\alpha_{2}},
$$

where $A$ is known as the "parameter of transformation" and $\alpha_{1}$ and $\alpha_{2}$ are constants to be determined. Under this transformation, Eq. (5) becomes

$$
d^{2} \bar{z} / d \bar{x}^{2}=n^{2} A^{2 \alpha_{1}-\alpha_{2}} \sinh \left(A^{\alpha_{2}} \bar{z}\right) .
$$


Equation (9) will be identical in form to Eq. (5) if we put

$$
\begin{gathered}
\alpha_{2}=0, \\
\bar{n}^{2}=n^{2} A^{2 \alpha_{1}-\alpha_{2}} .
\end{gathered}
$$

Equation (9) then gives

$$
d^{2} \bar{z} / d \bar{x}^{2}=\bar{n}^{2} \sinh (\bar{z}) .
$$

The next step in this method is to define the still-unknown initial slope to be equal to the parameter of transformation; i.e.,

$$
d z(0) / d x=A,
$$

which, upon transformation, gives

$$
A^{\alpha_{2}-\alpha_{1}}(d \bar{z}(0) / d \bar{x})=A .
$$

Since $\alpha_{1}$ is still an arbitrary constant, we may choose

$$
\alpha_{2}-\alpha_{1}=1
$$

to reduce Eq. (13) to

$$
d \bar{z}(0) / d \bar{x}=1
$$

Equations (10) and (14) give

$$
\alpha_{1}=-1, \quad \alpha_{2}=0 .
$$

The boundary condition at $\bar{x}=0$ (Eq. (6)) is transformed to

$$
\bar{z}(0)=0 .
$$

Finally, the parameter of transformation can be evaluated from the boundary condition at the second point (Eq. (7)) as

$$
A^{\alpha_{1}} \bar{x}=1: A^{\alpha_{2}} \bar{z}=\bar{n} A^{0.5 \alpha_{2}-\alpha_{1}}
$$

or

$$
\bar{x}=A: \bar{z}=\bar{n} A \text {. }
$$

Elimination of $A$ in Eq. (18) gives

$$
\bar{z}=\bar{n}(\bar{x}) \quad \text { (at the second point). }
$$

The solution of Eq. (5) then follows these steps: First, a value of $\bar{n}$ is assigned. Second, Eq. (11) is integrated as an initial value problem using initial conditions 
(15) and (17). Third, referring to Eq. (18), the integration in the second step is continued until Eq. (19) is satisfied. The value of $A$ at this point will be the parameter of the transformation, as shown in Eq. (18). Fourth, the corresponding value of $n$ can be calculated by Eq. (10). The process can be repeated by assuming another value of $\bar{n}$ and the solution of Eq. (5) for another value of $n$ will be obtained. By assuming a family of values of $\bar{n}$, a family of solutions for a family of values of $n$ can be generated. The only difference between the results from such a method as compared with those from iterative methods is that the values of $n$ are not equally spaced numbers, even though the values of $\bar{n}$ are. Table I shows

\section{TABLE I}

Selected Missing Initial Slopes, $d y(0) / d x$, for Various $\vec{n}$ s

\begin{tabular}{ccll}
\hline & & \multicolumn{2}{c}{$d y(0) / d x$} \\
\cline { 3 - 4 }$\tilde{n}$ & & \multicolumn{1}{c}{ This method } & \multicolumn{1}{c}{ Iterative } \\
\hline 21 & 4.956 & 0.04762 & 0.04761 \\
22 & 5.007 & 0.04545 & 0.04543 \\
55.74 & 6.000 & $0.17940\left(10^{-1}\right)$ & $0.17944\left(10^{-1}\right)$ \\
145.6 & 7.000 & $0.68681\left(10^{-2}\right)$ & $0.68695\left(10^{-2}\right)$ \\
386.85 & 8.001 & $0.25850\left(10^{-2}\right)$ & $0.25851\left(10^{-2}\right)$ \\
1037.0 & 9.001 & $0.99432\left(10^{-3}\right)$ & $0.99434\left(10^{-3}\right)$ \\
2790.6 & 9.999964 & $0.358346\left(10^{-3}\right)$ & $0.358347\left(10^{-3}\right)$ \\
$20,500.0$ & 12.003 & $0.48781\left(10^{-4}\right)$ & $0.48779\left(10^{-4}\right)$ \\
$150,000.0$ & 13.997 & $0.66667\left(10^{-5}\right)$ & $0.66675\left(10^{-5}\right)$ \\
\hline
\end{tabular}

some of the solutions for a range of values of $\bar{n}$. If the data are needed for plotting the missing slope as a function of $n$, the results such as those shown in Table I are sufficient. If tabulation of the missing slope for a few integer values of $n$ is desired, a simple Lagrange interpolation formula can be used for such purposes. Since only solutions for $n>5$ are of numerical interest, the results presented in Table I are in this range, even though the method can be applied to both small and large values of $n$.

To check the accuracy, the results are compared with those using an iteration method (Runge-Kutta forward integration by manually adjusting the missing initial slope on the terminal of the digit computer system). Such solutions are also shown in Table I (the last column). Also, in Table II, the solutions of $y$ and $d y / d x$ as functions of $x$ for $n=8$ are compared with the solutions by iteration. The agreement is excellent. 
TABLE II

Comparison with Iterative Solutions for $n=8$

\begin{tabular}{|c|c|c|c|c|}
\hline \multirow[b]{2}{*}{$x$} & \multicolumn{2}{|c|}{ This method } & \multicolumn{2}{|c|}{ Iterative } \\
\hline & $y$ & $d y ; d x$ & $y$ & $d y / d x$ \\
\hline 0.0 & 0.00000 & $0.25850\left(10^{-2}\right)$ & 0.00000 & $0.25851\left(10^{-2}\right)$ \\
\hline 0.2 & $0.76779\left(10^{-3}\right)$ & $0.66649\left(10^{-2}\right)$ & $0.76770\left(10^{-3}\right)$ & $0.66641\left(10^{-2}\right)$ \\
\hline 0.4 & $0.39585\left(10^{-2}\right)$ & $0.31778\left(10^{-1}\right)$ & $0.39581\left(10^{-2}\right)$ & $0.31775\left(10^{-1}\right)$ \\
\hline 0.6 & $0.19649\left(10^{-1}\right)$ & 0.15739 & $0.19649\left(10^{-1}\right)$ & 0.15739 \\
\hline 0.8 & $0.98566\left(10^{-1}\right)$ & 0.80923 & $0.98550\left(10^{-1}\right)$ & 0.80907 \\
\hline 1.0 & 1.00011 & $0.54611\left(10^{2}\right)$ & 1.00000 & $0.54605\left(10^{2}\right)$ \\
\hline
\end{tabular}

TABLE III

Comparison of Solutions by Various Methods

\begin{tabular}{|c|c|c|c|c|c|c|}
\hline \multirow[b]{2}{*}{$n$} & \multicolumn{2}{|c|}{ This method } & \multicolumn{2}{|c|}{ Miele et al. [6] } & \multicolumn{2}{|c|}{ Jones [5] } \\
\hline & $d y(0) / d x$ & $d y(1) d x$ & $d y(0) / d x$ & $d y(1) ; d x$ & $d y(0) / d x$ & $d y(1) / d x$ \\
\hline 5 & $0.45754\left(10^{-1}\right)$ & 12.000 & $0.45750\left(10^{-1}\right)$ & 12.1005 & $0.457\left(10^{-1}\right)$ & 一 \\
\hline 6 & $0.17940\left(10^{-1}\right)$ & 20.0072 & $0.17951\left(10^{-1}\right)$ & 20.0358 & - & - \\
\hline 10 & $0.35835\left(10^{-3}\right)$ & 149.168 & $0.35834\left(10^{-3}\right)$ & 148.4064 & $0.356\left(10^{-3}\right)$ & 148.4 \\
\hline
\end{tabular}

Table III compares the accuracies of $d y(0) / d x$ and $d y(1) / d x$ obtained by the present method with those presented by Miele, Aggarwal and Tietze [6] and by Jones [5]. Again, close agreement is obtained.

To conclude, the method offers a very effective alternative for problems of this type where solutions of the equation for a range of values of the parameter (in this problem, the parameter is $n$ ) are required.

\section{REFERENCES}

1. E. S. Weirel, Phys. Fluids. 2 (1959), 52.

2. B. A. Troesch, Intrinsic Difficulties in the Numerical Solution of a Boundary Value Problem, Internal Report NN-142, TRW Inc., Redondo Beach, Calif., 1960. 
3. L. Ehrlich, Experience with Numerical Methods for a Buundary Value Problem, Internal Report NN-141, TRW Inc., Redondo Beach, Calif., 1960.

4. S. M. Roberts and J. S. Shipman, J. Computational Phys. 10 (1972), 232.

5. D. J. Jones, J. Computational Phys. 12 (1973), 429.

6. A. Miele, A. K. Aggarwal, and J. L. Tietze, J. Computational Phys. 15 (1974), 117.

7. K. TÖPFER, Z. Math. Phys. 60 (1912), 397.

8. M. S. KLAMKIN, SIAM Ret. 4 (1962), 43.

9. T. Y. NA, SIAM Rev. 9 (1967), 204.

10. T. Y. NA, SIAM Rev. 10 (1968), 85.

ReCeIveD: January 22, 1975; REVISED April 9, 1975

J. P. CHIOU

T. Y. NA

Department of Mechanical Engineering The University of Michigan Dearborn, Michigan 48128 